\title{
Experimental investigation on surface characterization of chemical treated Natural fiber composite
}

\author{
C.Ramesh ${ }^{1}$, S.C.Prasanna ${ }^{2}$ \\ Associate professor, M.Kumarasamy College of Engineering Karur, Tamilnadu, India \\ hodmech@mkce.ac.in \\ Assistant Professor, M.Kumarasamy College of Engineering Karur, Tamilnadu, India \\ Prasannacareers89@gmail.com
}

\begin{abstract}
In this research work removal of lignin and hemicelluloses for untreated coir fiber reinforced composites was accomplished by suitable chemical treatment namely new method at optimum fiber loading such as $25 \%$ and $30 \%$. To avoid the problem of untreated coir fiber, chemical treatment was done. Final treated fiber extraction process was gone through new method. It results in treated cellulose composite at $25 \%$ and $30 \%$ lignin and hemicelluloses was found to be decreased was revealed in Fourier transform infrared spectroscopy analysis, it shows defibrillation, de-polymerization in treated composite was revealed in scanning electron microscopy analysis, and shows high oxygen content percentage than carbon content percentage about $22.06 \%$ which demonstrates removal of lignin in treated composite, was revealed in element detection analysis.. The main objective was accomplished by the removal of lignin and hemicelluloses of natural fiber through suitable chemical treatment and at optimum fiber loading.
\end{abstract}

\section{Indexing terms/Keywords}

Lignin, Hemicelluloses, Scanning Electron Microscopic, Fourier Transform Infrared Spectroscopy, Element Detection Analysis.

\section{Academic Discipline And Sub-Disciplines}

Mechanical Engineering- Materials - Natural fiber composite

\section{SUBJECT CLASSIFICATION}

Polymer composite

\section{TYPE (METHOD/APPROACH)}

Experimental Investigation

\section{INTRODUCTION}

Now a day's composites are used in wide variety of applications because of its constituent material can regain their original properties even after mixing. In polymers many research under gone through synthetic because of their promising properties. In this study natural Fiber reinforced polymer was investigated and chosen material was coir fiber because it is natural, low cost, low density, high specific strength and environmental friendly. The drawback of natural fiber has high moisture absorption capacity, and it consists large amount of lignin, hemicelluloses, pectin and wax which was amorphous in nature, due to which it possess low strength, and poor dynamic characteristics.. Thus in this study various chemical extraction processes was carried out namely New Method[1] and cross and Bevan method [2] to remove lignin, pectin, hemicelluloses content, reducing wear and to improve adhesion between fiber and matrix. Finally Treated cellulose fibril is extracted from New Method because large quantity of treated cellulose was obtained from new method, and in new method fiber was subjected to mild chemical treatment it does not affect cellulose content which tends to reduce lignin and hemicelluloses contents alone. Thus further extraction process gone through new method for 800 grams. After Extraction process obtained treated cellulose was 350 grams, for which composite sheets were made at different weight percentage such as $25 \%$ and $30 \%$ in case of both treated and untreated fiber by compression molding .To reduce moisture content and to improve strength reinforcement is done and matrix used for reinforcement is epoxy. For obtained composite sheets morphological analysis was carried out in scanning electron microscopic (SEM), for chemical composition analysis FT-IR (Fourier Transform Infrared Spectroscopy) analysis was carried out, and finally element detection analysis was carried out by EDX Technique to check weight percentage of carbon and oxygen. The microscopic analysis results at treated cellulose sample defibrillation, De-polymerization occurs, and material becomes soft comparison to untreated sample. In FT-IR absence of lignin and hemicelluloses was observed in both $25 \%$ and $30 \%$ of fiber loading, in element detection analysis it reveals that oxygen content is larger than carbon in case of treated composite ,it proves that amount of lignin content was reduced. Thus due to chemical treatment it demonstrates removal of lignin and hemicelluloses in case of treated cellulose composite at $25 \%$ and $30 \%$ of fiber loading comparison to untreated composite. The main theme of this research was to remove lignin and hemicelluloses content of untreated coir fiber reinforced composites through suitable chemical treatment namely new method [1] thus it results high removal Rate at $25 \%$ and $30 \%$ of treated cellulose composite.

\section{Experimental}

\section{Materials}


Coconut fiber is extracted from the outer shell of a coconut. Brown fibers are thick, strong and have high abrasion resistance. Density $1.4 \mathrm{~g} / \mathrm{cc}$ Single fiber, Breaking Elongation 30\%, Moisture regain at $65 \% \mathrm{RH} 10.5 \%$,

\section{Methods for the extraction of Treated cellulose}

In this research two extraction processes were carried out namely cross and Bevan method [2] and new method

\section{New Method}

Coir fiber is chopped for 3-4 mm length and it is soaked for $2 \%$ caustic soda $(\mathrm{NaOH})$ in the fiber liquor ratio of $1: 10$ and kept for $6 \mathrm{hrs}$ at a room temperature. Then fiber is washed several times with distilled wasted to remove sticking of $\mathrm{NaOH}$ in fiber thus weight reduction was achieved [1]. Steam exploded treatment is done for mercerized fiber for $1 \mathrm{hrs}$ around 200-250 degree Celsius in pressure Cooker thus removal of lignin content and hemicelluloses content is observed weight loss is Obtained after steam explosion. Then it is washed thoroughly with water, dried and weighted. During this process heat is applied around 200-250 degree Celsius [1]. Bleaching process is done for steam exploding Fiber using Naclo2 (Sodium chlorite) at ph. 2.3 [1]. The ph. of water is 7 is reduced to low ph at 2.3 value using Concentrated sulphuric acid (H2SO4) and by using sodium chlorite ph. is maintained at ph. 2.3. Heated for $1 \mathrm{hr}$. at $50-60$ degree .Then it is washed with distilled water to reduce the effect of sodium chlorite. Oxalic acid treatment is subjected to bleached fiber for mild acid treatment .Oxalic acid 5\% is taken for 800 grams of coir fiber and heated for $1 \mathrm{hrs}$ at $50-60$ degree followed by steam exploded treatment for $1 \mathrm{hrs}$ at 200-250 degree [1] maintained in pressure cooker. Then fibers are then washed thoroughly with water and dried. Thus due to mild chemical treatment cellulose content of fiber may not decrease [1]. Finally after acid treatment the dried fiber is subject to constant stirring process on Remi motor at constant speed of 200 rpm for further cellulose. Extracted treated cellulose from new method was shown in Figure 1.

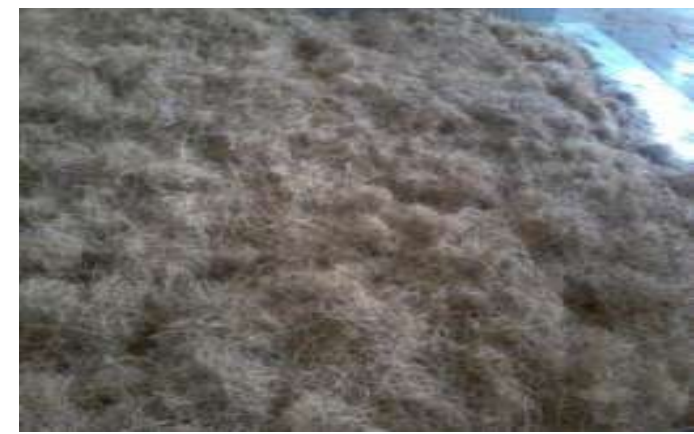

Fig 1. Final Stage Extracted Treated Cellulose after Stirring Process

\section{Cross and Bevan method}

In this step chopped coir fiber is first boiled with $1 \%$ of $\mathrm{NaOH}$ for $30 \mathrm{~min}$. after that it is washed with water until moist chlorine gas is removed and it is dried, weight reduction is achieved. Then after the fiber is treated with $2 \%$ of sodium sulphite and heated it for $5 \mathrm{~min}$. Finally it is washed with water and then it is dried and weighted.Fiber after sodium sulphite treatment is subjected to bleaching process by maintaining ph. at 4 thus ph. value is reduced using sulphuric acid and it heated with of potassium permanganate for $1 \mathrm{hrs}$. At 50-60 degree thus low molecular weight components partially get reduced. Thus it is washed with water again and again. Finally it is dried and weighted. The obtained treated cellulose from cross and Bevan method was shown in figure 2

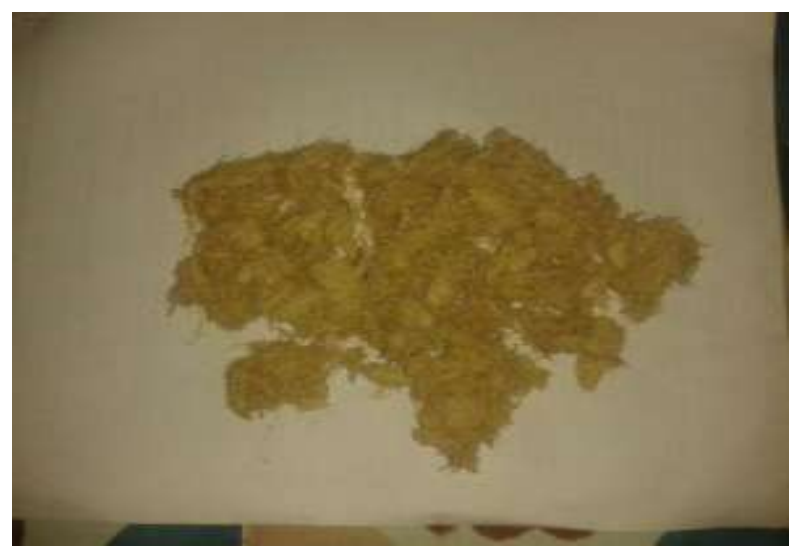

Fig 2 Obtained Treated Cellulose from Cross and Bevan method

\section{a. Composite sheet fabrication}

The matrix used in this process is epoxy because it is able to improve adhesion between fiber and matrix and low cost. The epoxy matrix and epoxy hardener is mixed in ratio of 10: 1 , in slow manner to avoid bubble formation in prolonged Duration [10]. The coir fiber of $25 \%$ and $30 \%$ of both treated and untreated by weight was spread over mold cavity. The 
fiber is placed in mold impression and mixture of resin and hardener is also placed in mold impression [10]. The other half of mold plate is placed to complete whole setup. Force is applied at the rate of 2 ton per square inch which squeezes the fiber and resin mixture which is able to take mold impression shape and it is kept at constant pressure at 85-95 degree for 3-4 hrs [10]. And then it is cooled for $9-12$ degree for $4-5 \mathrm{hrs}$, to avoid hardener effect in composite plate [10]. After the whole process is completed sheets are withdrawn from mold impression [10]. The fabricated composite sheets were shown in Figure 3 \& 4 .
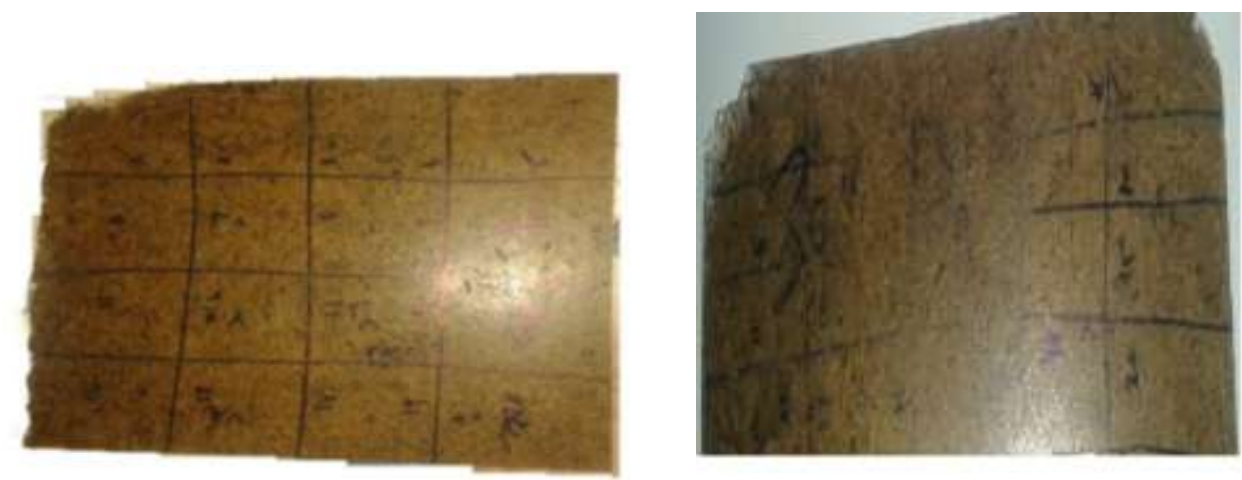

Fig 3 Untreated Composite Sheets

Fig 4 Treated Composite Sheets

\section{b. Scanning Electron Microscopic(SEM)}

Morphological analysis of samples at different weight percentage such as $25 \%$ and $30 \%$ in case of both treated and untreated composite were evaluated by scanning electron microscope Carl Zeiss EVO 18 at $20 \mathrm{Kv}$. The samples are made for $1 \mathrm{~mm} \times 1 \mathrm{~mm} \times 3 \mathrm{~mm}$.for the prescribed size of sample morphology analysis were carried out for both treated and untreated composite.

\section{c. Fourier Transform Infrared Spectroscopy(FT-IR)}

Fourier transform infrared spectroscopy (FT-IR) of the samples was recorded by an IR-tracer 100 Schimadzu spectrophotometer. About $1 \mathrm{~mm} \times 1 \mathrm{~mm}$ sample was placed into small particles of liquid nitrogen. The samples were mixed with kbr and pressed into small disc about $1 \mathrm{~mm}$ thickness.

\section{d. Element Detection Analysis}

Energy-dispersive X-ray spectroscopy (EDS, EDX, or XEDS), sometimes called energy dispersive X-ray analysis (EDXA) or energy dispersive X-ray microanalysis (EDXMA), is an analytical technique used for the elemental analysis or chemical characterization of a sample. It relies on an interaction of some source of excitation. In this study element detection analysis were carried out for both treated and untreated sample at both $25 \%$ and $30 \%$ weight in the dimension of $1 \mathrm{~mm} x$ $1 \mathrm{~mm} \times 3 \mathrm{~mm}$ sample size. Element detection analysis was carried out in SEM along With EDX technique to determine chemical composition in which able to determine weight percentage of carbon and oxygen.

\section{Results and Discussion}

\section{A.Scanning Electron Microscopy(SEM)}

The results showed in Fig $5 \& 6$ as untreated raw sample, and chemically treated fiber sample. It shows that rough surface and little porous in case of untreated raw sample at both $25 \%$ and $30 \%$ weight of fiber loading, and shows strong binding of cementing components lignin and hemicelluloses in untreated raw with average diameter of $100 \mu \mathrm{m}$ in case of $25 \%$ untreated and $30 \%$ untreated samples. In general alkali treatment at $2 \%$ remove lignin, and hemicelluloses content in fiber further steam explosion and bleaching process removes lignin, hemicelluloses, pectin, and tannin from inner part of fiber by De-polymerization and defibrillation, lignin oxidized by bleaching agent which allows to lignin degradation that leads to formation three functional groups such as hydroxyl, carbonyl, and carboxylic that access lignin to be soluble in alkali solution. From the above study it demonstrates that in untreated raw sample it reveals rough surface, porous and shows impurities such as lignin and hemicelluloses at both $25 \%$ and $30 \%$ of weight in Fig 5 . In case of treated composite it demonstrates removal of lignin and hemicelluloses due to alkali treatment followed by stream explosion, bleaching and oxalic acid treatment it allows to defibrillation and De-polymerization that reveals removal of lignin, hemicelluloses, wax and pectin that contained in fiber. Each elementary fiber shows a compact structure; exhibiting an alignment in the fiber axis direction with some non-fibrous components in the fiber surface. It shows that chemically treated fiber shows removal of lignin and hemicelluloses with micro fibrils average diameter around 20-200 $\mu \mathrm{m}$ shown in Fig 6 


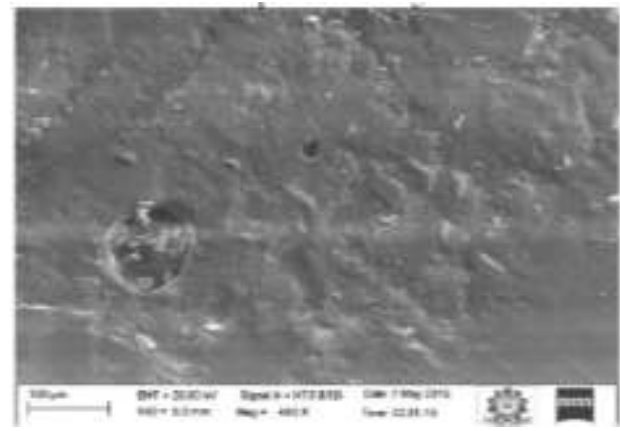

Fig 5.Untreated coir fiber SEM Image

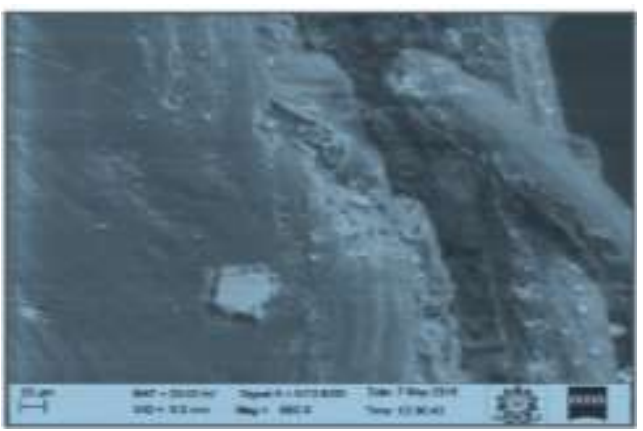

Fig 6. Treated coir fiber SEM Image

\section{B) Fourier Transform Infrared Spectroscopy(FT-IR)}

The results shows that lignin and hemicelluloses content wave number was absent in case of both treated cellulose at $25 \%$ and $30 \%$ weight comparison to untreated composites. The composite material is composed of alkaline, esters, ketone, alcohol; aromatics with different oxygen functional group same as observed in literature. An infrared red transmittance spectrum with some main observed peaks for different weight percentage in case of both treated and untreated was shown in table 1. All samples should be dry because it is difficult to extract due to cellulose water interaction.

Table1: Infrared Transmittance spectrum (\%) for the different weight percentage in case of both treated and untreated composite.

\begin{tabular}{|l|l|l|l|l|}
\hline $\begin{array}{l}\text { FT-IR } \\
(\mathrm{cm}-1) \\
\text { Spectra } \\
(\% \mathrm{~T})[1]\end{array}$ & $\begin{array}{l}\text { O-H } \\
\text { Stretch } \\
{[1]}\end{array}$ & $\begin{array}{l}\text { C-H } \\
\text { Vibration } \\
{[1]}\end{array}$ & $\begin{array}{l}\text { C=O } \\
\text { Stretch } \\
{[1]}\end{array}$ & $\begin{array}{l}\text { Absorbed } \\
\text { water [1] }\end{array}$ \\
\hline $\begin{array}{l}25 \% \\
\text { Untreated }\end{array}$ & 3969.5 & 2731.2 & 1753.29 & 1608.63 \\
\hline $\begin{array}{l}30 \% \\
\text { Untreated }\end{array}$ & 3975.2 & 1865.17 & 1755.22 & 1610.56 \\
\hline $\begin{array}{l}25 \% \\
\text { Treated }\end{array}$ & 3980.9 & 2106.27 & - & 1608.46 \\
\hline $\begin{array}{l}30 \% \\
\text { Treated }\end{array}$ & 3983.3 & - & - & 1600.34 \\
\hline
\end{tabular}

From the above table1 shows that the characteristics peak wave number $1753.2-1755.22 \mathrm{~cm}-1$ is responsible for hemicelluloses and lignin content and it was presented in both untreated composite at $25 \%$ and $30 \%$ weight. At chemical treated composite it was chiefly absent in both weight percentage. The wave number from $1600-1690 \mathrm{~cm}-1$ is responsible for moisture absorption capacity. It shows that increasing to decreasing range from untreated to treated sample at different weight percentage in the range $1610.56-1600.34 \mathrm{~cm}-1$.

Table 2: Infrared Transmittance spectrum (\%) for the different weight percentage in case of both treated and untreated composite.

\begin{tabular}{|l|l|l|l|}
\hline $\begin{array}{l}\text { FT-IR }(\mathrm{cm}-1) \\
\text { Spectra }(\% T)[1]\end{array}$ & $\begin{array}{l}\text { C-H } \\
\text { Stretching } \\
{[1]}\end{array}$ & $\begin{array}{l}\text { Aromatic } \\
\text { ring } \\
\text { Vibration } \\
\text { of lignin } \\
{[1]}\end{array}$ & $\begin{array}{l}\text { S-C } \\
{[1]}\end{array}$ \\
\hline $25 \%$ Untreated & 1365.60 & - & 1060.13 \\
\hline $30 \%$ Untreated & - & 1276.88 & 1053.10 \\
\hline $25 \%$ Treated & - & - & 1068.13 \\
\hline $30 \%$ Treated & - & - & 1072.42 \\
\hline
\end{tabular}

From the above table 2 shows that the characteristics peak wave number $1200-1390 \mathrm{~cm}-1$ is responsible for hemicelluloses and lignin content and another possibility carboxyl adsorption, it was presented in both untreated composite at $25 \%$ weight and aromatic ring vibration was presents in $30 \%$ untreated. At chemical treated composite it was chiefly absent in both weight percentage. The wave number from 1000-1080 cm-1 is responsible for cellulose to cellulose interaction. It shows that decreasing range to increasing range from untreated to treated sample at different weight 
percentage in the range $1053.10-1072.42 \mathrm{~cm}-1$. From FT-IR we can conclude that lignin and hemicelluloses binding components were removed in chemical treatment during $\mathrm{NaOH}$ treatment it breaks hydrogen bond, thus it reduces $\mathrm{OH}$ Concentration, further steam explosion, bleaching process it leads to formation of three function groups that access lignin and hemicelluloses to be soluble in chemical treatment, thus raw fiber have this characteristics peak $1700-1760 \mathrm{~cm}-1$ and $1200-1390 \mathrm{~cm}-1$ chiefly responsible for lignin and hemicelluloses. Above results were shown as graph with respect to wave number Vs Infrared transmittance (\%), in Fig 7 \& 8.

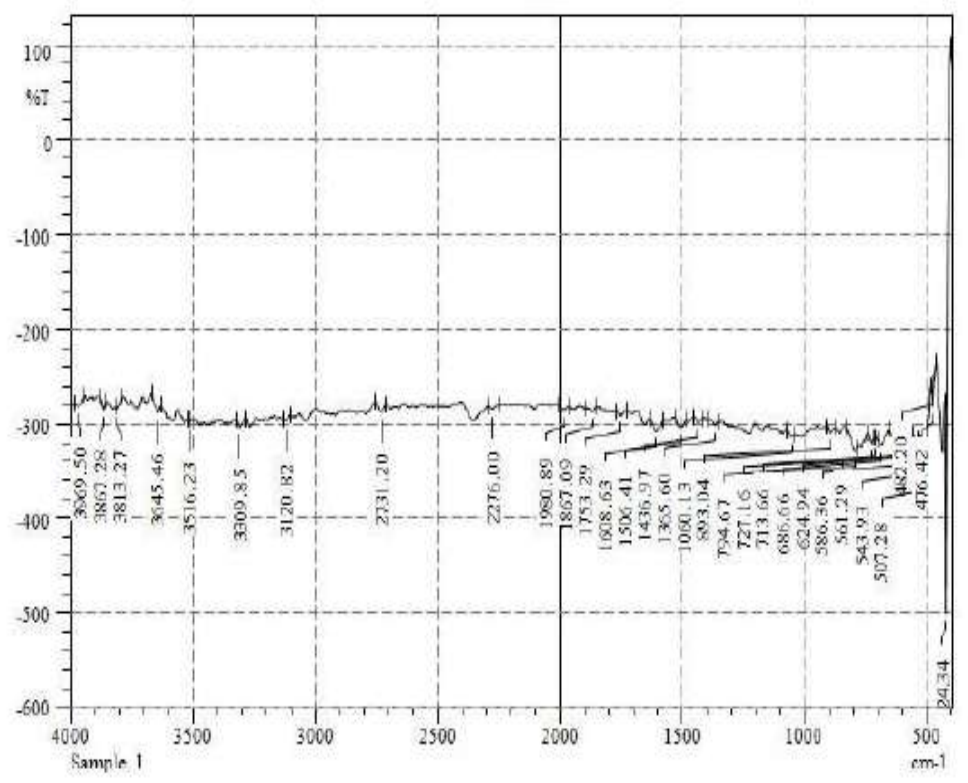

Fig 7 Graph Shows For Untreated 25\% Weight Sample Chemical Composition With Respect To Infrared Transmittance

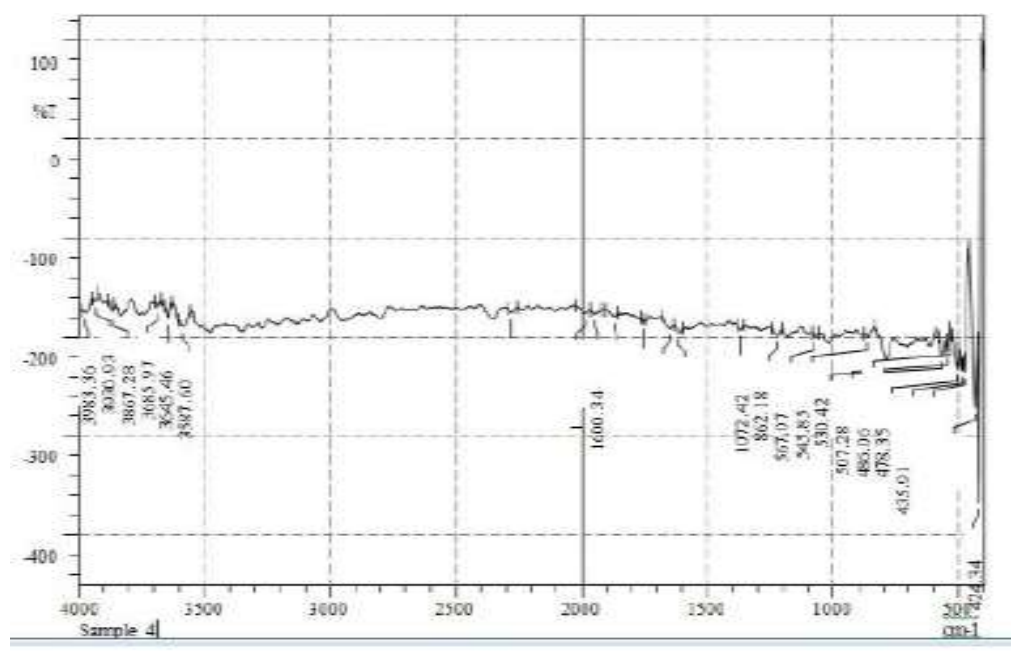

Fig 8 Graph Shows For Treated 30\% Weight Sample Chemical Composition With Respect To Infrared Transmittance (\%)

\section{C) Element Detection Analysis}

The results shows that at untreated sample at $25 \%$ and $30 \%$ weight of fiber loading it shows higher carbon content weight percentage than oxygen it clearly demonstrates due to higher lignin content, because lignin which was aromatic in nature with high carbon content comparison to cellulose and hemicelluloses. Thus during chemical treatment cellulose will be isolated from impurities which shows higher weight percentage of oxygen than carbon content. At $25 \%$ and $30 \%$ of fiber loading higher weight percentage of oxygen was observed at $30 \%$ of treated sample, but both $25 \%$ and $30 \%$ of treated sample demonstrates lignin content elimination and results higher oxygen percentage comparison to untreated samples at both $25 \%$ and $30 \%$ weight. The results of weight percentage of oxygen and carbon content was shown in Fig 8 . 


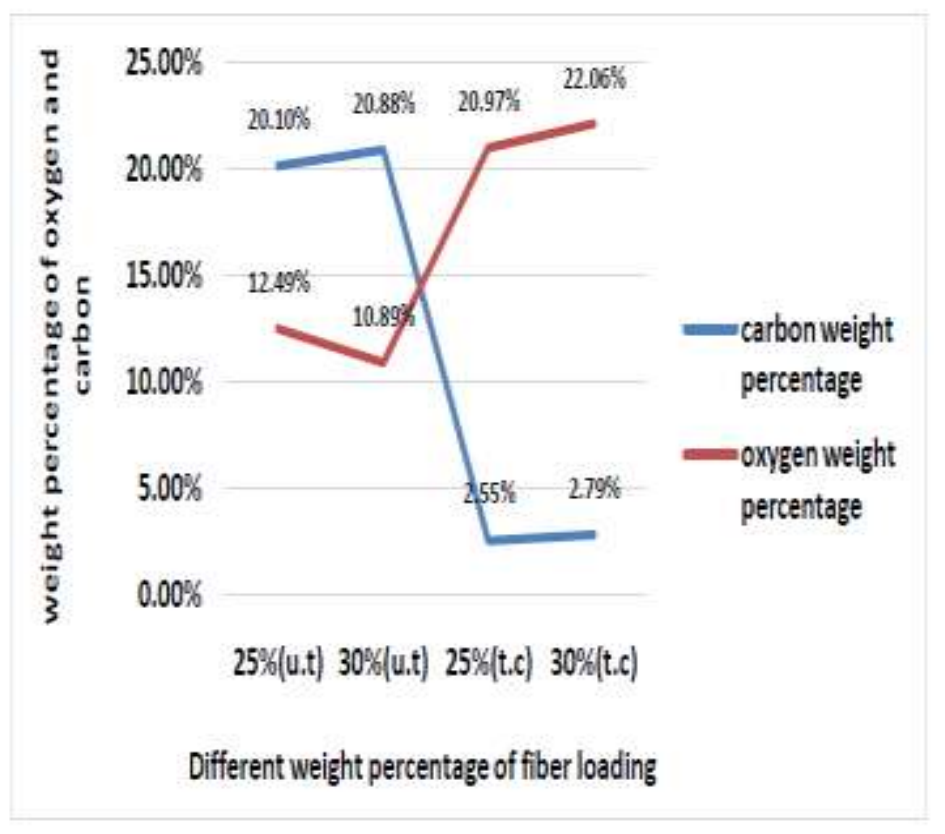

Fig 8. Weight Percentage of Carbon and Oxygen Vs Fiber Loading

\section{Conclusion}

The main theme of this research was to remove lignin and hemicellulose content of untreated coir fiber reinforced composites and it was accomplished by suitable chemical treatment namely new method thus it possess low amplitude level, and high damping factor in treated composites at both $25 \%$ and $30 \%$ of fiber loading. Microscopic Analysis Such as Scanning Electron Microscopic, Fourier Transform Infrared Spectroscopy, and Element Detection Analysis were Carried out for Treated and Untreated Composite, it Shows Defibrillation And Depolymerization in Case of Treated Cellulose at $25 \%$ and $30 \%$ was recorded in SEM. The Absence of Lignin, Hemicelluloses, Pectin, and Some wax Content was observed in Treated Cellulose at $25 \%$ and $30 \%$ Comparison to Untreated. In Element Detection Analysis Weight Percentage of Oxygen is higher than Carbon at $30 \%$ of Treated Cellulose, Secondly at $25 \%$ of Treated Cellulose Comparison to Untreated Composites. So we conclude that removal of lignin and hemicelluloses was observed in Case of Treated Composite Comparison to Untreated Composite at $25 \%$ and $30 \%$ of weight. In future scope at $30 \%$ of treated composite, damping characteristics can be enhanced.

\section{References}

1. E.Abraham, "Extraction of Nanocelluose Fibril from Lignocelluloses Fibril a Novel Approach"-.Carbohydrate Polymers, Vol. 86, and pp: 1468-1475, 2011

2. Hrishkeshchattopadhyay, "New Method for the Estimation Cellulose"-Method for the Estimation of Cellulose, Vol. 12, and pp: 23-44, 1945.

3. Juan.I.moran,"Extraction of Cellulose and Preparation of Nanocelluose from Sisal Fiber"- Cellulose, Vol.15, and pp: 149-159, 2008.

4. João Paulo SaraivaMoraisa "Extraction and Characterization of Nanocelluose Structures from Raw Cotton Linter"Carbohydrate Polymers, Vol.91, and pp: 229-235, 2012.

5. A.I.S. Brígida, "Effect of Chemical Treatments on Properties of Green Coconut Fiber"- Carbohydrate Polymers, Vol.79, and pp: 832-838, 2010.

6. N.Rajini,"Dynamic Mechanical Analysis and Free Vibration Behavior in Chemical Modifications of Coconut Sheath/Nano-Clay Reinforced Hybrid Polyester Composite"- Journal of Composite Materials,Vol.47, and pp: 3105-3121, 2013.

7.C.Ramesh" Fabrication of ammcs by using stir casting method for hand lever"- International Journal of New Technologies in Science and Engineering, Vol. 2, Issue. 1, 2015, ISSN 2349-0780

8. A.Z.Ahmadmujahid, "Experimental Modal Analysis (EMA) On Coconut Coir Fiber Reinforced Composite"- Global Engineers and Technologist Review,pp: 15-20, 2011.

9. N.Vijayasai, "Investigation on Dynamic Behaviour of Hybrid Sisal/Bagasse Fiber Reinforced Epoxy Composites" International Journal of Innovative Research in Advanced Engineering, Vol. 1, and pp: 357-369, 2014.

10. Priyasinghal, "Effect of Various Chemical Treatments on the Damping Property of Jute Fiber Reinforced Composite" International Journal of Advanced Mechanical Engineering, Vol. 4, and pp: 413-424, 2014. 
11. Merra, "Experimental and Numerical Investigation on Dynamic Behaviors of Beam for Different Cross Section"Department of civil engineering, NIT thesis,2013.

12. I.Z.Bujang, "Study on the Dynamic Characteristic of Coconut Fiber Reinforced Composites" - Noise, Vibration and Comfort Research Group, pp: 185-202, 2007.

13. Weiqun GU, "Interfacial Adhesion Evaluation of Uniaxial Fiber-Reinforced-Polymer Composites by Vibration Damping of Cantilever Beam" - Materials Engineering Science Program, Doctor of Philosophy Thesis, 1997.

14. N.Rajini, "Effect of Lamina Fiber Orientation on Tensile and Free Vibration (by Impulse Hammer Technique) Properties of Coconut Sheath/ Sansevieriacylindrica Hybrid Composites" -Advanced Material Research, Vol. 984, and pp: 172-177, 2015.

15. Alexander Bismarck, "Surface Characterization of Flax, Hemp and Cellulose Fiber; Surface Properties and the Water Uptake Behaviour" -Polymer Composites, Vol. 23, and pp: 872-894, 2002.

16. Charles W, "Compression Molding, ASM Handbook" -Composites, Vol. 21, and pp: 516-535, 2001

17. S.Jayabal,"Effect of soaking time and concentration of $\mathrm{NaOH}$ solution on mechanical properties of coir-polyester composites"- Indian Academy of Sciences, Vol.35 pp: 567-574, 2012.

18. N.Rajini,"Mechanical and Free Vibration Properties of Montmorillonite Clay Dispersed with Naturally Woven Coconut Sheath Composite"- Journal of reinforced plastics and composites,Vol.31 (20), pp: 1364-1376, 2012.

19. C.Ramesh "Comparison of coatings based on nano and commercial Titanium Dioxide as pigment on mild steel substrates"-International Journal of Emerging Researches in Engineering Science and Technology, Volume 2, Issue 6, July'15

20. H. Salmah, "Treated Coconut Shell Reinforced Unsaturated Polyester Composites"- International Journal of Engineering \& Technology, Vol.13 pp: 94-103, 2013.

21. K. Senthilkumar, "Influence of Orientation on Tensile and Flexural Properties of Sisal Fiber Polyester Composite"Journal of Chemical and Pharmaceutical Sciences, pp: 172-174, 2015.

22. H.Todd Robert, "Manufacturing Processes Reference Guide"- New York: Industrial P, Incorporated, pp: 219-220, 1993.

23. P.A.Udaykumar, "Studies On Effects of Short Coir Fiber Reinforcement on Flexural Properties of Polymer Matrix"International Journal of Research in Engineering and Technology, Vol 3, pp: 37-41, 2014.

24."Beginners Guide to Machine Vibration”- commestinstruments.LTD, 2006.

25.“Dewesoft FRF user manual 7.1”,www.dewesoft.com,- Version 1.0, May 2014.

26.P.Ganesa, "Free Vibration Behaviour of Glass Fiber Reinforced Composite"-Middle East Journal of Scientific Research, Vol 20(6), pp: 734-737, 2014.

\section{Author' biography with Photo}

\begin{tabular}{|l|l|}
\hline & $\begin{array}{l}\text { Mr.C.Ramesh works as Associate professor at M.Kumarasamy } \\
\text { College of Engineering, Karur, Tamilnadu, India in the Department of } \\
\text { Mechanical Engineering. He holds an undergraduate degree in } \\
\text { Mechanical Engineering and a master's degree in Computer aided } \\
\text { design. He has submitted his Ph.D. thesis on solar energy. Having } \\
17 \text { years of academic experience. }\end{array}$ \\
\hline & $\begin{array}{l}\text { Mr.S.C.Prasanna works as Assistant Professor at M.Kumarasamy } \\
\text { College of Engineering, Karur, Tamilnadu, India in the Department of } \\
\text { Mechanical Engineering. He holds an undergraduate degree in } \\
\text { Mechanical Engineering and a master's degree in Manufacturing } \\
\text { Engineering. He has } 4 \text { years of academic experience. }\end{array}$ \\
\hline
\end{tabular}

